CASE REPORT

\author{
M. Chow \\ C. McDougall \\ C. O'Kelly \\ R. Ashforth \\ E. Johnson \\ D. Fiorella
}

\section{Delayed Spontaneous Rupture of a Posterior Inferior Cerebellar Artery Aneurysm Following Treatment with Flow Diversion: A Clinicopathologic Study}

SUMMARY: In this report, we present the fatal spontaneous delayed rupture of a previously unruptured large PICA aneurysm following treatment with the PED. Pathology at postmortem examination has supported the theory that intra-aneurysmal thrombus may acutely destabilize the aneurysm wall. Aneurysms with an anatomic arrangement that promote continued flow into the neck may not be optimal candidates for the flow-diversion treatment strategy.

\begin{abstract}
ABBREVIATIONS: $\mathrm{AAA}=$ abdominal aortic aneurysm; $\mathrm{AS}=$ anterior spinal artery; $\mathrm{B}=$ basilar; $\mathrm{HE}=$ hematoxylin-eosin; ILT = intraluminal thrombus; LAIC = left anterior inferior cerebellar artery; LV = left vertebral artery; $M=$ medulla; $M R A=M R$ angiography; $P=$ pons; $P E D=$ Pipeline Embolization Device; PICA = posterior inferior cerebellar artery; $\mathrm{pO}_{2}=$ partial pressure of oxygen; $\mathrm{POD}=$ postoperative day; $\mathrm{RPIC}=$ right posterior inferior cerebellar artery; $\mathrm{RV}=$ right vertebral artery
\end{abstract}

$\mathbf{F}$ low-diverting stents are a new therapeutic option for the endovascular treatment of complex intracranial aneurysms. ${ }^{1-3}$ During the initial experience, these devices have been applied with remarkable success to achieve a definitive treatment of previously untreatable vascular lesions. Their recent approval for commercial sale in Europe, Asia, and Canada has thus resulted in a more widespread implementation of the technology. Predictably, as our experience with these devices continues to evolve, the utility, the limitations and potential complications of the flow-diversion strategy are becoming evident. ${ }^{4-6}$ It is important to report and attempt to understand the successes as well as the inevitable complications that arise during its application to accelerate our understanding of this new technology. To this end, we report a complication related to the application of a flow diverter (PED; ev3, Irvine, California) used to achieve the reconstruction of a giant posterior fossa aneurysm. We discuss the potential mechanisms for this treatment failure.

\section{Case Report}

\section{Presentation}

The index patient (an adult woman) came to the attention of the neurosurgical consult service after a CT scan of the head demonstrated a very large aneurysm in the region of the left vertebral artery. Although discovered incidentally, detailed questioning revealed a history of progressive impairment of ambulation with gait instability and difficulty swallowing during the past several months. On physical examination, the patient had subtle leftward tongue deviation on pro-

Received November 14, 2010; accepted after revision December 13.

From the Division of Neurosurgery, Department of Surgery (M.C., C.M., C.O.), Department of Diagnostic Imaging (R.A.), and Department of Laboratory Medicine and Pathology (E.J.), University of Alberta, Edmonton, Alberta, Canada; and Division of Cerebrovascular and Endovascular Neurosurgery (D.F.), Department of Neurosurgery, State University of New York, Stony Brook, New York.

Please address correspondence to Michael Chow, MD, Division of Neurosurgery, Department of Surgery, University of Alberta, Edmonton, AB, Canada; e-mail: Michael.Chow@ albertahealthservices.ca

http://dx.doi.org/10.3174/ajnr.A2532 trusion, a positive Hoffman reflex, and an upgoing plantar reflex on the right.

MRA and digital subtraction angiography studies revealed a wideneck 23-mm left vertebral artery aneurysm (Fig 1). The neck of the aneurysm encompassed a 10-mm segment of the left vertebral artery. The proximal aspect of the base of the aneurysm incorporated the left PICA. The right vertebral artery was hypoplastic. The aneurysm did not incorporate the vertebrobasilar junction.

\section{Treatment Considerations}

Because of the poor natural history of this very large and progressively symptomatic aneurysm, treatment was recommended. Surgical clipping, though likely to provide a durable exclusion of the lesion, was thought to be both technically challenging and associated with a high risk of operative morbidity. Conventional endosaccular coiling of the aneurysm, while also technically challenging, was thought to be unlikely to provide a durable exclusion of the aneurysm and, more importantly, could not be achieved without accepting an occlusion of the origin of the PICA. Moreover, coil embolization would not alleviate the posterior fossa mass effect created by the aneurysm sac. Prior experience had indicated that curative reconstruction with the PED was typically associated with a regression of aneurysmal mass effect. For these reasons, endovascular reconstruction with the PED was offered as a potentially more straightforward and physiologic option for treatment. After consideration of these options, the patient provided informed consent for treatment with the PED. At this time, the PED was not yet commercially available in Canada. This patient's treatment was performed under a Health Canada Special Batch Access provision to allow the use of an unapproved device.

\section{Procedure}

The patient was pretreated with both aspirin and clopidogrel. The procedure was performed under full intravenous heparinization with an activated clotting time maintained between 250 and 300 seconds throughout the procedure. The microcatheters were delivered through a 6F Envoy guide catheter (Cordis, Miami Lakes, Florida), which had been positioned within the distal cervical segment of the left vertebral artery. Traversing the wide neck of the aneurysm proved to be challenging. To achieve access across the aneurysm neck, we had 


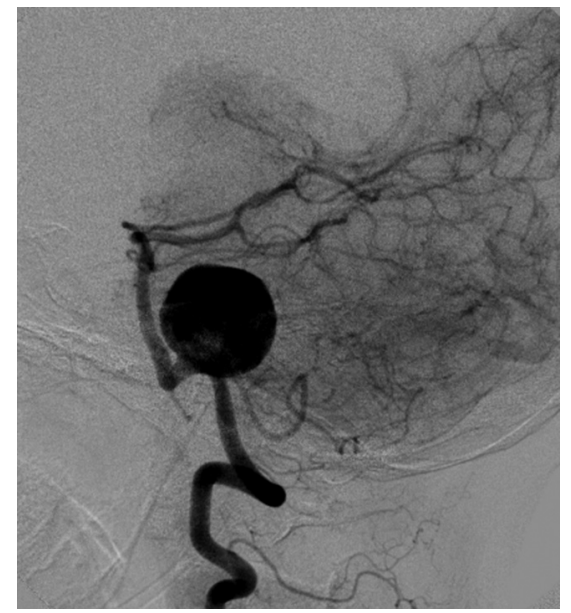

Fig 1. Lateral projection from angiography performed from a catheter positioned within the left vertebral artery demonstrates a very large $(23-\mathrm{mm})$ wide-neck aneurysm arising from the mid-V4 segment of the vertebral artery. The aneurysm circumferentially incorporates a 10-mm-length segment of the parent artery. The PICA arises from the proximal aspect of the aneurysm neck. The neck of the aneurysm does not incorporate the vertebrobasilar junction.

to loop a 0.010 -inch X-Pedion microwire (ev3) around the fundus of the aneurysm to achieve an angle sufficient to allow the microwire to egress from the aneurysm neck into the more distal vertebral-basilar system. Subsequent straightening of the wire was achieved by manipulating a $4 \times 7 \mathrm{~mm}$ HyperForm balloon (ev3) over the microwire, inflating the balloon within the basilar trunk, and then gently pulling the slack out of the proximal balloon microcatheter. The balloon catheter was then exchanged over a 300-cm 0.010-inch X-Celerator exchange wire (ev3) for an Excelsior SL-10 microcatheter (Boston Scientific, Natick, Massachusetts). The Excelsior SL-10 microcatheter was then exchanged for a Marksman delivery microcatheter (ev3) over a $300 \mathrm{~cm} 0.014$ inch X-Celerator exchange microwire and advanced into the midbasilar artery.

A single $4 \times 20 \mathrm{~mm}$ PED was then deployed, with the distal aspect of the construct positioned within the proximal basilar artery and the proximal aspect terminating within the V4 segment of the left vertebral artery, proximal to the aneurysm neck. Following placement of the PED, control angiography demonstrated contrast stasis within the aneurysm that persisted into the venous phase (Fig 2). No complications were observed during or immediately after the procedure.

\section{Postoperative Course}

Due to the concern over the potential for acute thrombosis of the aneurysm with an abrupt increase in local mass effect or the induction of perianeurysmal inflammation, the patient was started on dexamethasone and observed in the neurointensive care unit for 3 days after the procedure. The patient did not develop any new neurologic deficits during the perioperative period and was discharged home on POD 5. Steroids were tapered during the hospitalization and discontinued at the time of discharge. The patient remained on dual antiplatelet therapy.

Three days after discharge (POD 8), she returned to the emergency department with severe intractable headache. She was neurologically stable and was admitted to the neurosurgical service. An MR imaging study showed that the aneurysm had progressed to nearcomplete thrombosis. MRA (Fig 3A) demonstrated a tiny niche of residual flow within the proximal aspect of the aneurysm neck, which supported the patency of the left PICA, which originated from this

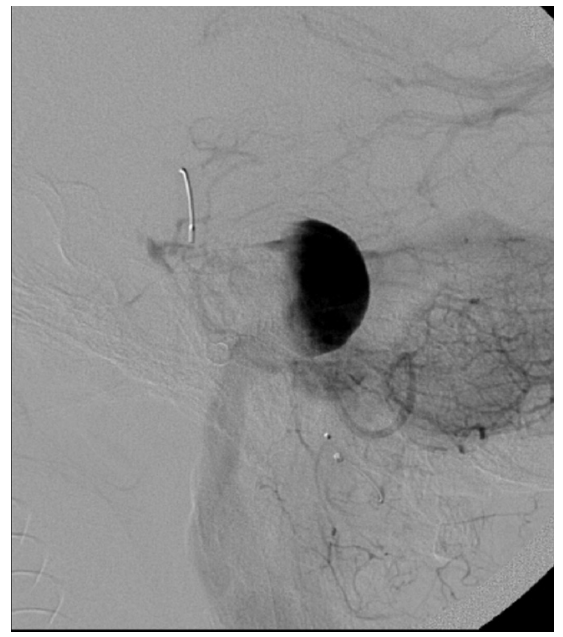

Fig 2. Lateral projection from an angiogram obtained immediately after successful deployment of a single $4 \times 20 \mathrm{~mm}$ PED construct across the aneurysm neck. Contrast material is stagnant within the aneurysm fundus, persisting into the venous phase of angiography. Contrast material was noted to washout from the aneurysm fundus into the PICA, which also remained faintly opacified throughout the venous phase.

aspect of the aneurysm. The left vertebral, basilar, and PICA arteries all remained patent. The degree of local mass effect and brain stem compression as well as the amount of regional T2 signal-intensity abnormality within the adjacent brain stem remained unchanged. No hydrocephalus had developed. Note was made of some linear enhancement of the aneurysm wall (Fig $3 B$ ). The patient was discharged home 2 days following admission (POD 10) with some improvement in her headache.

Ten days following discharge (POD 20), the patient suddenly collapsed at home. On arrival at an outside hospital, she was found to have a Glasgow Coma Scale score of 3 with fixed and dilated pupils. A CT scan (Fig 4) revealed subarachnoid hemorrhage as well as parenchymal hemorrhage within the brain stem extending from the dome of the aneurysm. The aneurysmal hemorrhage had dissected through the brain stem into the ventricular system. She was transferred to a tertiary hospital intensive care unit where she was determined to be brain dead. The patient's family consented to a postmortem examination.

\section{Pathology}

Postmortem examination of the brain confirmed rupture of the aneurysm via its dome buried in the pontomedullary junction, directly opposite to and remote from the stent-bridged aneurysmal neck (Fig 5). The associated hemorrhage had dissected into and through the length of the brain stem into the third ventricle, distending it and the lateral ventricles with blood.

Microscopic examination showed the lumen of the aneurysm to be occupied by thrombus that evoked a brisk resorptive reaction at the base (Fig 6) but little from the lateral walls and dome. Through the thrombus track zones of fresh blood coalescing into a stream along a wall pointing to the rupture site. Although there was no inflammation at this site in the proximity of the dome, there were foci of mural necrosis with loss of fibroblasts, mural hyalinization, and infiltrates of acute inflammatory cells, with or without dissecting microhemorrhages. In some locales, these microhemorrhages tracked into the subarachnoid space and were of various ages, suggestive of repeated episodes of bleeding. This mural necrosis would predispose to aneurysmal rupture. 

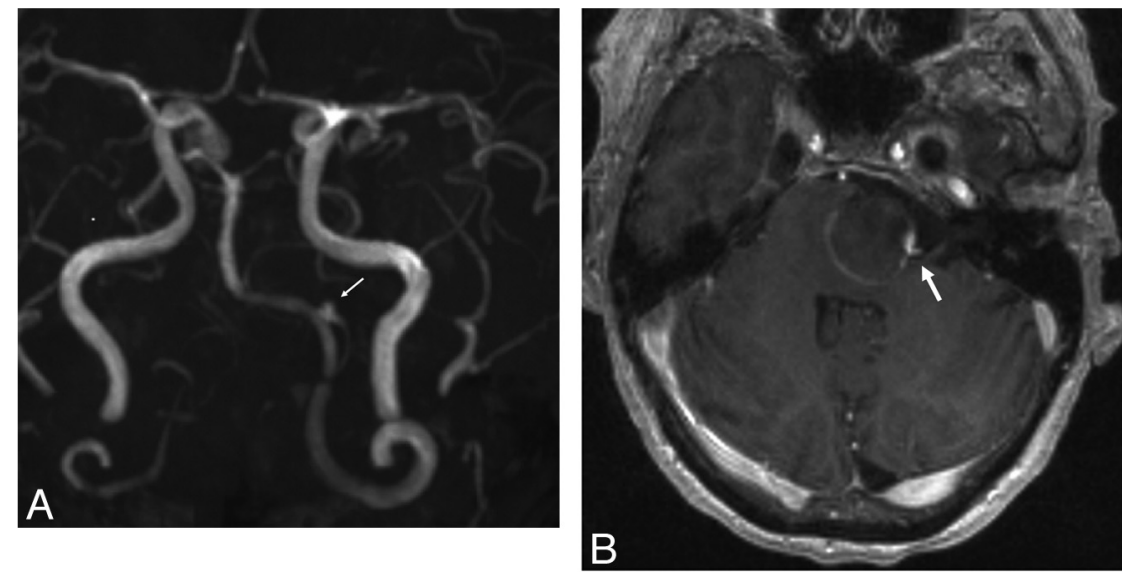

Fig 3. A, Maximum intensity projection from an MRA performed on POD 9 demonstrates near-complete thrombosis of the aneurysm fundus. The left PICA remains patent, filling from a tiny niche of residual flow in the region of the aneurysm neck (arrow). $B$, Axial postcontrast T1-weighted sequence demonstrates linear enhancement of the aneurysm wall marginating the intra-aneurysmal thrombus. A focal niche of enhancement corresponds to the small neck remnant, which allows the patency of the PICA (arrow).

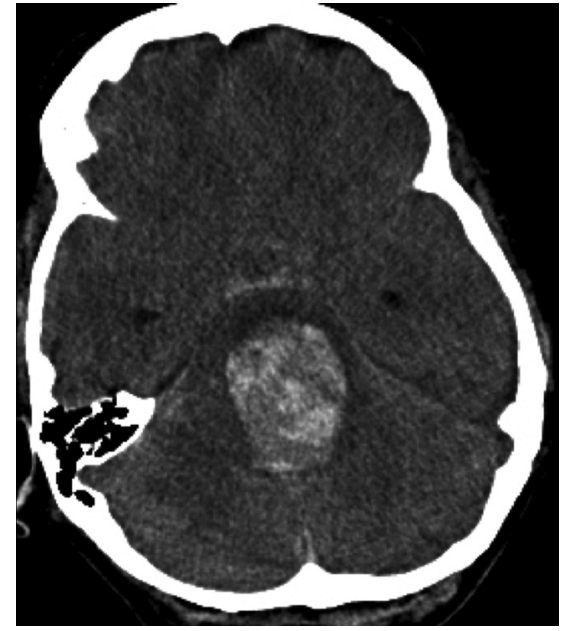

Fig 4. Noncontrast head CT scan obtained on POD 20 demonstrates aneurysmal rupture with dissection through the brain stem and into the fourth ventricle. A small amount of subarachnoid hemorrhage is noted within the prepontine cistern.

\section{Discussion}

The present case example demonstrates the delayed fatal rupture of a previously unruptured cerebral aneurysm after a technically successful treatment with the PED. The lesion was nearly completely thrombosed just before the documented rupture with only a tiny niche of residual flow near the origin of the PICA, where it arose from the aneurysm neck. Gross and microscopic inspection of the aneurysm at postmortem examination did not demonstrate rupture in the region of the PICA origin, where intra-aneurysmal flow was preserved, but remote from this residual focus of aneurysm filling at the distal aspect of the fundus.

Thus, we are presented with the paradoxical situation of delayed aneurysm rupture occurring after a technically successful treatment, with the rupture point demonstrated to arise from a segment of the aneurysm fundus that appeared to be "protected" by a large volume of intervening intra-aneurysmal thrombus. A number of similar cases of postprocedural rupture have recently been reported in the literature. ${ }^{5,6}$ However, the mechanism by which aneurysm rupture may occur after flow diversion remains unknown.

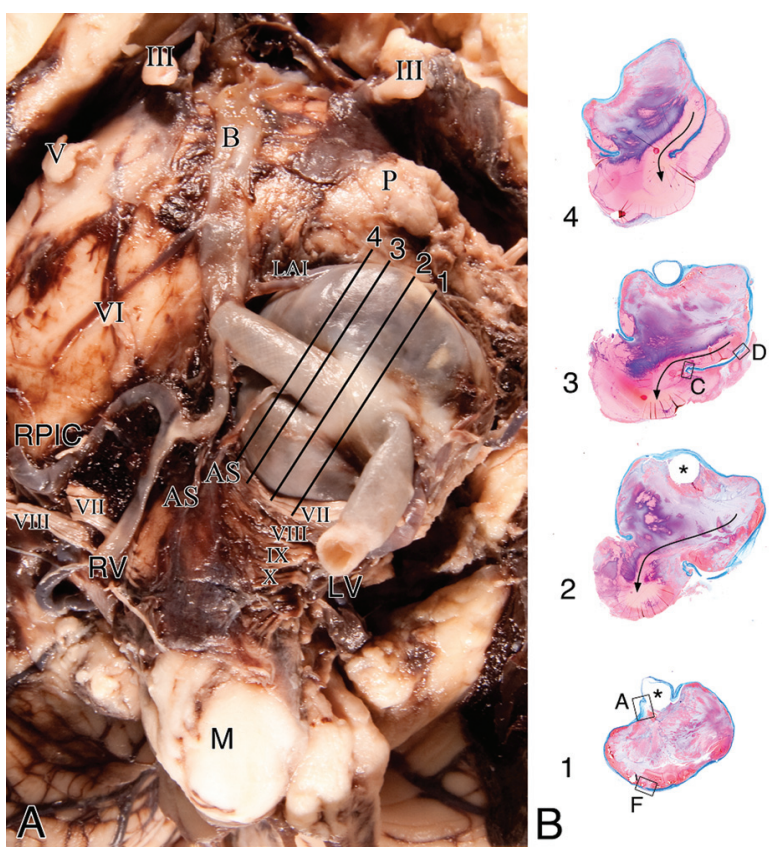

Fig 5. $A$, Base of the brain stem showing a large saccular aneurysm arising from the midportion of the left vertebral artery and embedded in the cerebellopontine angle, compressing the ipsilateral cranial nerves VII and VIII. Note that postdissection remnants of acute subarachnoid hemorrhage surround the aneurysm. Note distention of the segment of the artery at the base of the aneurysm by the stent. Cranial nerves are designated by Roman numerals. Lines with numbers indicate the plane of section of whole mounts of the aneurysm and vertebral artery as illustrated in $B$ (Masson trichrome). $B$, Cross-sections of the aneurysm show incorporation of the vertebral artery into its wall (levels 1 and 2) and exit (level 1). Location of the stent (asterisk) is outlined by the luminal thrombus that partially fills the aneurysm. Fresh blood tracks along a lateral wall to the rupture through the dome (arrow). Boxes with letters represent microscopic fields shown in Fig 6.

The index case illustrates the following points:

1) Evolving intra-aneurysmal thrombus may not provide protection from aneurysm rupture. On the contrary, evolving thrombosis may actually cause a transient destabilization of the aneurysm wall, increasing rather than decreasing its propensity for rupture.

2) Any residual filling of aneurysms treated with a "standalone" flow-diversion strategy may preserve the potential for aneurysm rupture.

3) Aneurysms with anatomic configurations that could re- 

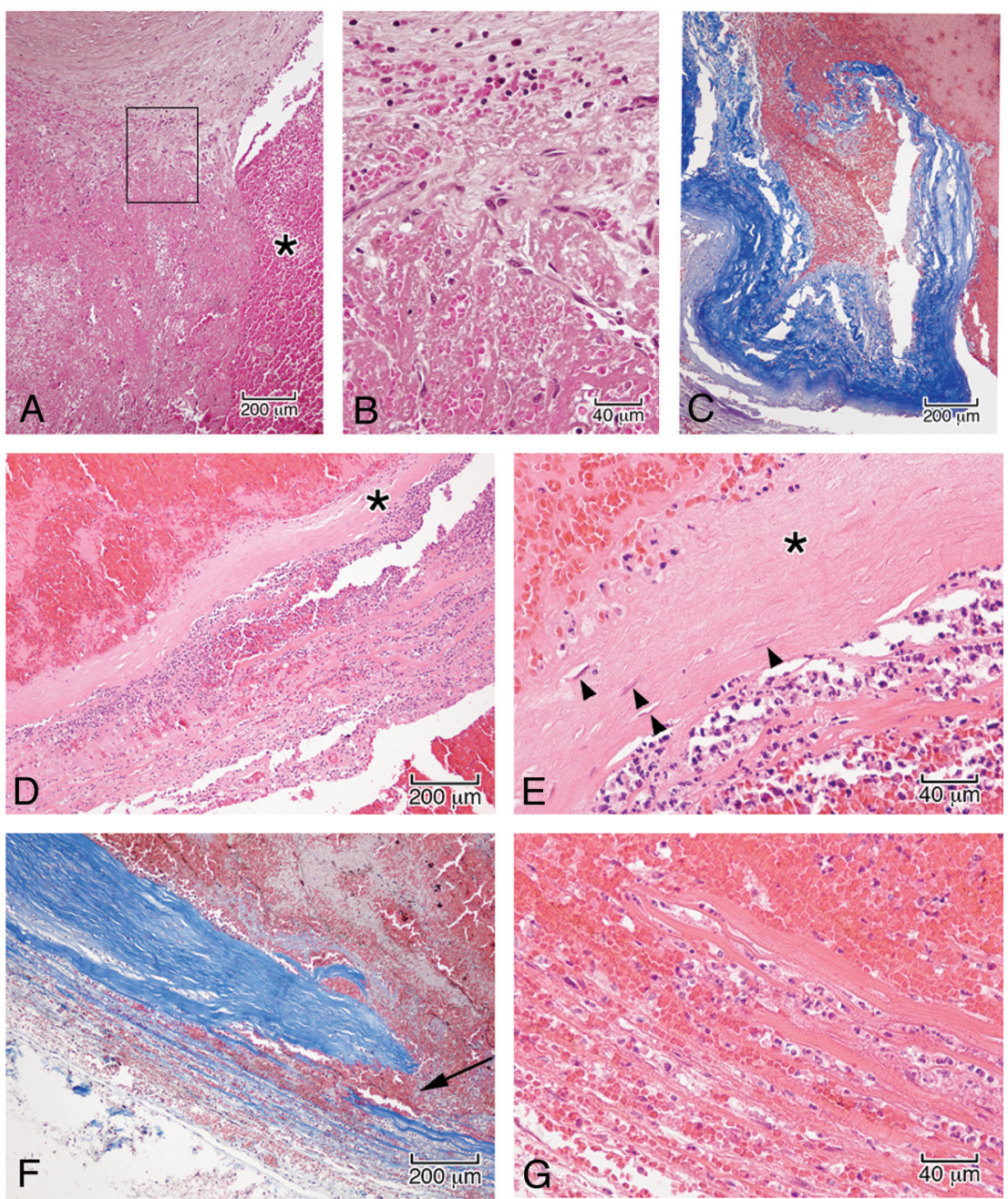

Fig 6. Microscopic features in the wall of the aneurysm corresponding to the boxed regions in Fig $5 B$. $A$, Junction of the arterial wall with the aneurysm, showing mural thrombus adjacent to fresh blood in the ostium of the aneurysm (asterisk). B. High power of the area in the box demonstrates organization of the thrombus by sprouts of capillaries ( $A$ and $B$, hematoxylin-orcein-phloxine-saffron). $C$, Site of rupture shows frayed tearing of the wall, suggesting dislodgment of any focal mural necrosis by the hemorrhage (Masson trichrome). $D$, Segment of the wall displays focal attenuation (asterisk) and necrosis with attenuated infiltrates of acute inflammatory cells along the outer meningeal portion of the wall. $E$, High power of the wall shows focal loss of fibroblasts (asterisk); arrowheads indicate remaining fibroblasts ( $D$ and $E, H E$ ). $F$, In other regions of the wall, dissecting microhemorrhage (arrow) is associated with the mural necrosis. $G$, Micro-hemorrhage associated with mural necrosis seen in high power. (F, Masson trichrome; $G, H E)$.

tard progression to complete thrombosis (eg, aneurysms arising from bifurcations or aneurysms with incorporated branch vessels) are probably suboptimal candidates for the flow-diversion strategy.

We considered each of these points within the context of our evolving understanding of the flow-diversion strategy and provide hypotheses about the mechanism by which delayed rupture may occur.

\section{Evolving Intra-Aneurysmal Thrombus May Cause a Transient Destabilization of the Aneurysm Wall, Increasing, Rather Than Decreasing its Propensity for Rupture}

Intuitively, one would predict that partial thrombosis of a cerebral aneurysm would actually function in a protective manner, insulating the aneurysm wall from direct contact with blood flow and thereby reducing the forces (like wall shear stress) that could contribute to aneurysm growth or rupture. There are, however, to our knowledge, no longitudinal studies looking at the natural history of partially thrombosed large saccular intracranial aneurysms because they are relatively rare lesions and they are usually treated aggressively after identification.

AAAs, while in many ways different from cerebral aneurysms, potentially represent an analogous scenario to the progressively thrombosing or partially thrombosed very largegiant intracranial aneurysm. Thus, the existing knowledge about AAAs may potentially provide valuable insight into the index case. ${ }^{7-18}$

Our understanding of the evolution and effects of thrombus in AAAs is far more developed than our understanding of the same process in large-giant cerebral aneurysms for the following reasons:

1) AAAs are more common than very large-giant intracranial aneurysms.

2) AAAs frequently harbor a considerable volume of ILT at the time of diagnosis, while most cerebral aneurysms do not.

3) Unlike very large or giant cerebral aneurysms, unruptured AAAs are often managed conservatively and observed with serial noninvasive imaging (CT or sonography). As such, 
there is a very well-developed imaging literature documenting the behavior of AAAs with time.

4) Histopathologic specimens from autopsies or obtained during the surgical repair of AAAs are far more plentiful than are those from very large-giant cerebral aneurysms.

Effect of ILT Burden on AAA Growth and Rupture. Although several studies have suggested that ILT reduces the shear stress on the wall of an AAA, ${ }^{9}$ the preponderance of clinical data indicate, paradoxically, that increasing volumes of ILT are associated with accelerated rates of aneurysm growth and an increased propensity for aneurysm rupture. ${ }^{7-14}$

Stenbaek et $\mathrm{al}^{7}$ showed that the accumulation of ILT on serial CT was a stronger predictor of aneurysm rupture than overall aneurysm growth. In fact, in this clinical series, none of the AAAs without ILT went on to rupture; all ruptures occurred in the subset of lesions that contained some in situ thrombus. Similarly, Satta et $\mathrm{al}^{12}$ showed that the thickness of ILT within an AAA was associated with an increased risk of rupture. Vorp et $\mathrm{al}^{8}$ showed that a larger ILT burden is associated with an accelerated pace of AAA growth. A global consideration of these data led Speelman et $\mathrm{al}^{9}$ to conclude that the reduction in shear stress produced by ILT was not protective from the more dominant effect of negatively impacting the integrity of the aneurysm wall and ultimately perpetuating AAA growth and rupture.

Mechanism by Which ILT Might Contribute to Aneurysm Growth and Rupture: Mural Hypoxia, Inflammation, and Enzymatic Degradation. The nutritional vessels of the aortic wall supply oxygen to the aortic adventitia and outer media, while the intima and inner media are supplied by luminal blood. ${ }^{11}$ Vorp et $\mathrm{al}^{8}$ hypothesized that ILT contributes to hypoxia of the aneurysm wall, resulting in neovascularization, inflammation, and a loss of structural integrity. ${ }^{11,13,14}$ In a series of patients, $\mathrm{pO}_{2}$ levels in the aneurysm wall covered by thicker ILT $(>4 \mathrm{~mm})$ were on average $18 \%$ of the luminal value, while $\mathrm{pO}_{2}$ levels within the wall covered by little or no $(<4 \mathrm{~mm})$ ILT were $60 \%$ of the luminal value. ${ }^{8}$ Tissue in the thicker ILT group also showed a greater number of inflammatory cells. The aneurysm wall in patients with thicker ILT had less tensile strength. The authors hypothesized that the increased concentration of inflammatory cells resulted in an increase in the local proteolytic activity. Moreover, they cited data indicating that inflammatory cells, particularly macrophages, exposed to hypoxic conditions may have enhanced bioreactivity and increased elastase production, resulting in an environment that promotes an enzymatic degradation of the aneurysm wall adjacent to the ILT. ${ }^{15}$

The histologic analysis of the present case revealed a remarkable similarity to these observations, with inflammatory infiltrates, mural thinning, and frank mural necrosis within aneurysm wall adjacent to the endoluminal thrombus.

How Does Rupture Occur through a Region of the Aneurysm That Appears Thrombosed and is Theoretically Protected from Blood Flow? CT and sonographic studies of AAAs have demonstrated that rupture may be precipitated by penetration or bleeding into the thrombus mass, with rupture subsequently occurring in the subjacent thrombus-covered wall. ${ }^{16-18}$ This breach of the ILT by flowing blood may be demonstrated on CT as a "high-attenuating crescent sign." ${ }^{16}$ Histopathologic correlation of specimens from patients demon- strating a high-attenuating crescent sign on CT demonstrated small nonendothelialized clefts with seeping blood penetrating through fragile points within the inner layer of the mass of the ILT. These clefts were thought to progressively merge and thus expand to create larger channels of free-flowing intraluminal blood within the outer layer of the ILT. These coalescent clefts lead imminently to hemorrhage within and ultimately through the fragile degraded aneurysm wall.

As our pathologic analysis revealed, a similar process may occur within partially thrombosed cerebral aneurysms if some intra-aneursymal flow is preserved after treatment. In the index case, a coalescent cleft was observed to have developed along the lateral wall of the aneurysm, leading to the rupture point at the dome.

\section{Any Residual Filling of Aneurysms Treated with a Flow- Diversion Strategy May Preserve the Potential for Aneurysm Rupture}

Thus, we hypothesize that intracranial aneurysms may behave in a way very similar to AAAs following the induction of thrombosis after flow diversion. We propose that evolving intra-aneurysmal thrombus functions to destabilize the aneurysm wall via mechanisms of hypoxia, inflammation, and enzymatic degradation while lacking the structural integrity to adequately shield the aneurysm wall from the systemic blood flow. We theorize that flow diverters, like the PED, when used without coils, create a race between 2 simultaneous processes-progression to complete aneurysm thrombosis and thrombosis-induced destabilization of the aneurysm wall. Potentially, this may result in either of 2 outcomes:

1) Aneurysm cure. In most cases, the aneurysm completely thromboses, there is no residual intra-aneurysmal flow, and the neck becomes covered by a neoendothelial-intimal layer, which effectively seals off the aneurysm. Once isolated from the cerebrovasculature, the aneurysm no longer carries with it a potential for rupture. At this point, the intra-aneurysmal thrombus mass gradually undergoes regression.

2) Persistent intra-aneurysmal flow with maintained potential for progressive growth and/or rupture. In rare cases, intra-aneurysmal flow persists for a longer time. The hypoxic, inflammatory, and proteolytic effects of the evolving thrombus on the aneurysm wall result in a loss of structural integrity. The persistence of intra-aneurysmal flow preserves the potential for the continued accumulation of intra-aneurysmal thrombus and aneurysm growth. Similarly, any amount of intra-aneurysmal flow may penetrate into the evolving thrombus mass and gain access to the destabilized aneurysm wall, potentially leading to precipitous aneurysm rupture (as in the index case).

Thus, in some cases, a partially thrombosed aneurysm after flow diversion may be considerably more dangerous than in its previously untreated nonthrombosed state. Correspondingly, it seems that near-complete occlusion after conventional coil embolization has an entirely different implication than does near-complete thrombosis after flow diversion. While smallneck remnants after coiling have a well-described generally benign natural history, the same is not likely true for aneurysms treated with flow diversion as a stand-alone technology. 


\section{Aneurysms with Anatomic Configurations That Could} Retard Progression to Complete Thrombosis are Probably Suboptimal Candidates for the Flow-Diversion Strategy

The instability of the aneurysm-thrombus mass may be prolonged by any factors that preserve intra-aneurysmal flow. There are several potential etiologies commonly encountered:

1) Anatomic features of the target aneurysm that perpetuate intra-aneurysmal flow after flow diversion:

A) Aneurysms with branches arising from the neck or fundus (such as in the index case with the incorporation of PICA into the proximal aneurysm fundus)

B) Aneurysms that incorporate a bifurcation (eg, basilar apex, carotid terminus).

2) Characteristics of the flow-diverting construct that do not promote efficient complete aneurysm occlusion:

A) Suboptimal device design

B) Technically inadequate deployment of the construct.

At this stage, it is probably prudent to avoid aneurysms with anatomic configurations that might promote continued aneurysmal filling after flow diversion. It is also important also to realize that once a flow-diverting construct is built across the neck of an aneurysm, endovascular access to the lesion is lost indefinitely and options for retreatment are essentially limited to the placement of additional flow diverters or parent artery deconstruction.

\section{Conclusions}

Delayed aneurysm rupture may occur days to weeks after the application of flow-diverting devices to treat intracranial aneurysms. We hypothesize that this phenomenon may be attributable to destabilizing effects of evolving acute thrombus on the aneurysm wall. This destabilization persists as long as there is any residual flow within the aneurysm fundus. Any anatomic arrangement that perpetuates intra-aneurysmal flow (eg, branches arising from the aneurysm fundus) may prolong this period of destabilization, exposing the patient to an increased risk of aneurysm rupture. Aneurysms with such anatomic features are not optimal for treatment with flow diverters.

Disclosures: Cian James O'Kelly: Other Financial Relationships: Proctor, Details: served as proctor for Pipeline cases; David Fiorella: Research Support (including provision of equipment or materials): NIH, Siemens Healthcare, Details: NIH, salary support for SAMMPRIS Trial; Siemens Healthcare, research grants. State University of New York-Stony Brook Cerebrovascular, Speaker Bureau: Micrus-Codman-JnJ, MicroVention, ev3, Details: MicrusCodman-JnJ: consulting, significant conflict $(>\$ 10,000)$, MicroVention Terumo: consult- ing, significant conflict $(>\$ 10,000)$, ev3: paid consultant $(>\$ 10,000)$, Pipeline proctor (reimbursed for travel expenses only). Ownership Interest: ReVasc Inc, Details: acquired by Micrus-Codman-JnJ: intellectual property, patents, royalties, significant conflict $(>\$ 10,000)$.

\section{References}

1. Fiorella D, Kelly ME, Albuquerque FC, et al. Curative reconstruction of a giant midbasilar trunk aneurysm with the Pipeline embolization device. Neurosurgery 2009;64:212-17, discussion 217

2. Fiorella D, Woo HH, Albuquerque FC, et al. Definitive reconstruction of circumferential, fusiform intracranial aneurysms with the Pipeline embolization device. Neurosurgery 2008;62:1115-20, discussion 1120-21

3. Lylyk P, Miranda C, Ceratto R, et al. Curative endovascular reconstruction of cerebral aneurysms with the Pipeline embolization device: the Buenos Aires experience. Neurosurgery 2009;64:632-42, discussion 642-43, quiz N636

4. Fiorella D, Hsu D, Woo HH, et al. Very late thrombosis of a Pipeline embolization device construct: case report. Neurosurgery 2010;67:E313-14, discussion E314

5. Kulcsar Z, Houdart E, Bonafé A, et al. Intra-aneurysmal thrombosis as a possible cause of delayed aneurysm rupture after flow-diversion treatment. AJNR Am J Neuroradiol 2011;32:20-5. Epub 2010 Nov 11

6. Cebral J, Mut F, Raschi M. Aneurysm rupture following treatment with flowdiverting stents: computational hemodynamics analysis of treatment. AJNR Am J Neuroradiol 2011;32:27-33. Epub 2010 Nov 11

7. Stenbaek J, Kalin B, Swedenborg J. Growth of thrombus may be a better predictor of rupture than diameter in patients with abdominal aortic aneurysms. Eur J Vasc Endovasc Surg 2000;20:466-69

8. Vorp DA, Lee PC, Wang DH, et al. Association of intraluminal thrombus in abdominal aortic aneurysm with local hypoxia and wall weakening. $J$ Vasc Surg 2001;34:291-99

9. Speelman L, Schurink GW, Bosboom EM, et al. The mechanical role of thrombus on the growth rate of an abdominal aortic aneurysm. J Vasc Surg 2010;51: $19-26$

10. Dobrin PB, Baker WH, Gley WC. Elastolytic and collagenolytic studies of arteries: implications for the mechanical properties of aneurysms. Arch Surg 1984;119:405-09

11. Vorp DA, Federspiel WJ, Webster MW. Does laminated intraluminal thrombus within abdominal aortic aneurysm cause anoxia of the aortic wall? J Vasc Surg 1996;23:540-41

12. Satta J, Laara E, Juvonen T. Intraluminal thrombus predicts rupture of an abdominal aortic aneurysm. J Vasc Surg 1996;23:737-39

13. Vande Geest JP, Wang DH, Wisniewski SR, et al. Towards a noninvasive method for determination of patient-specific wall strength distribution in abdominal aortic aneurysms. Ann Biomed Eng 2006;34:1098-106

14. Vorp DA, Wang DH, Webster MW, et al. Effect of intraluminal thrombus thickness and bulge diameter on the oxygen diffusion in abdominal aortic aneurysm. J Biomech Eng 1998;120:579-83

15. Albina JE, Henry WL Jr, Mastrofrancesco B, et al. Macrophage activation by culture in an anoxic environment. J Immunol 1995;155:4391-96

16. Arita $\mathrm{T}$, Matsunaga $\mathrm{N}$, Takano $\mathrm{K}$, et al. Abdominal aortic aneurysm: rupture associated with the high-attenuating crescent sign. Radiology 1997;204: 765-68

17. Mehard WB, Heiken JP, Sicard GA. High-attenuating crescent in abdominal aortic aneurysm wall at CT: a sign of acute or impending rupture. Radiology 1994;192:359-62

18. Catalano O, Siani A. Ruptured abdominal aortic aneurysm: categorization of sonographic findings and report of 3 new signs. J Ultrasound Med 2005;24: 1077-83 